\title{
Activités de formation des migrants en France : des transitions expansives?
}

\author{
Aurélie Beauné \\ Docteure de l'université Paris Descartes (laboratoire EDA), \\ Qualifiée en sciences de l'éducation et sciences du langage \\ Membre de l'équipe éditoriale d'Adjectif et du eJournal
}

AURELIEBEAUNE@HOTMAIL.COM

Résumé

Nous présentons ici les principaux résultats d'une recherche de thèse ${ }^{1}$ portant sur les processus à l'œuvre pour les activités de formation des migrants en France : suite à une importante vague de réformes, comment évoluent ces activités? Peut-on constater, au sens de la théorie de l'apprentissage expansif, une expansion de l'objet de ces activités?

Nous avons observé l'activité de trois organismes de formation accueillant des publics migrants, sur une durée d'un an pour l'un d'entre eux et de six mois pour les deux autres. Les données recueillies dans ces trois organismes (observations et entretiens) ont permis de produire des synthèses à l'issue de différents cycles d'observation. Ces synthèses ont ensuite été soumises aux acteurs pour être discutées collectivement. L'ensemble de ces données a donné lieu à plusieurs analyses des contradictions pour chaque système d'activité et pour l'ensemble des trois systèmes considérés.

Les analyses menées ont permis d'identifier une évolution spontanée des activités que nous avons nommée " transition restrictive » et qui peut être engendrée par la coexistence des quatre dimensions structurelles de tout système d'activité.

Mots-clés : Formation des migrants; Théorie de l'apprentissage expansif; Théories de l'activité; Interventions formatives.

1. Dirigée par G.-L. Baron et M. Bento, intitulée « Contribution à l'étude des pratiques instrumentées des formateurs d'adultes : le cas du Diplôme Initial de Langue Française (DILF) », cette thèse de doctorat de sciences de l'éducation a été soutenue publiquement le 20/11/15 à l'université Paris Descartes. 


\section{Contexte}

Nous avons débuté une recherche relative aux pratiques instrumentées ${ }^{2}$ de formation des adultes migrants en France en juillet 2011 : dans un contexte de réformes des conditions d'accueil et de formation des adultes migrants, la problématique explorée avait trait aux utilisations de TIC pour la formation des migrants et, en particulier, de ceux non ou faibles lecteurs.

Trois mois après, soit en octobre 2011, un label et un agrément " Français Langue d'intégration » $(\mathrm{FLI})^{3}$ ont été créés par décret : cet événement a accentué notre intérêt pour l'étude de l'évolution des règles relatives à la formation des adultes migrants.

À l'instar d'autres labels existants pour l'enseignement du Français comme Langue Étrangère (FLE), l'obtention du label ou de l'agrément FLI via un audit permet dorénavant aux organismes de formation de :

— «délivrer une attestation aux apprenants migrants, qui sera reconnue par l'administration française pour les procédures d'accès à la nationalité française et de résidence en France »;

— «d'accéder aux subventions publiques de l'État et éventuellement aux subventions des collectivités territoriales » (Adami et al., 2011, p. 6).

Une polémique a suivi leur création (Puren, 2014), les détracteurs du FLI reprochant notamment une ingérence du politique au sein des décisions didactiques. Ces deux épisodes évoquent les soubresauts qui affectent l'histoire des activités de formation des migrants ou les « bouleversements, changements soudains et reprises » ${ }^{4}$ du développement psychologique dans une conception vygotskienne (Kozulin, 1986, p. 104).

L'étude de la littérature disponible à propos de ces activités nous a tout d'abord permis de re-contextualiser ces événements récents au sein des évolutions du cadre institutionnel pour la formation des migrants, en France, depuis les années 1960. Parmi les multiples décisions gouvernementales, actions de la société civile (associations, collectifs) ou des syndicats, nous avons pu distinguer au moins trois grandes périodes dans l'histoire des activités de formation des migrants, périodes que nous présentons brièvement dans la suite.

2. Ce syntagme réfère, en sciences de l'éducation, aux utilisations de technologies informatisées pour l'enseignement et l'apprentissage; les théories de l'activité conçoivent généralement que toutes les pratiques sont instrumentées.

3. On a cherché à utiliser le moins possible de sigles et d'acronymes, en nombre foisonnant dans le champ des activités de formation des migrants.

4. Traduction libre de «Vygotsky perceived psychological development as a dynamic process full of upheavals, sudden changes, and reversals. This process, however, ultimately leads to the formation of the cultural, higher mental functions ». 


\subsection{Développement historico-culturel des activités de formation des migrants : une synthèse}

Les activités de formation des migrants s'inscrivent dans la continuité de l'évolution historico-culturelle de celles d'apprentissage, telle qu'analysée par Engeström (1987, pp. 108-117). Elles relèvent plus précisément d'activités formelles d'apprentissages, institutionnalisées avec ceux scolaires dans le cadre de la formation initiale, puis avec ceux de la formation continue des adultes.

Leur histoire spécifique ne semble pouvoir commencer qu'à partir des années 1960, époque à partir de laquelle on peut parler $\mathrm{d}^{\prime}$ " alphabétisation de travailleurs migrants » sans commettre d'anachronisme :

La formation linguistique des migrants constitue [...], dans les années 60, un secteur peu structuré, très peu professionnalisé, faiblement subventionné par les pouvoirs publics et touchant une population nombreuse au regard des besoins langagiers objectifs (Leclercq, 2012, p. 177).

Une lente structuration de ce champ de pratiques s'observe jusqu'à la création des lois françaises relatives à la formation continue de 1971 (De Lescure, 2004).

La crise économique résultant des chocs pétroliers a ensuite influencé cette structuration, ouvrant une deuxième grande période dans l'histoire des activités de formation des migrants : nous retenons principalement le renforcement des liens entre formation des migrants et insertion professionnelle, ainsi que des dispositions relatives à la fermeture des frontières (Leclercq, 2012).

La troisième période importante s'ouvre dans les années 1990 et il semble qu'elle perdure encore actuellement. En effet, à cette époque, "sur fond d'analyses récurrentes démontrant les limites qualitatives persistantes des formations dispensées aux migrants [... ] » (Leclercq, 2012, p. 183), une orientation nouvelle pour le financement de ces activités est décidée via la constitution de marchés publics comportant plusieurs appels d'offres, ce qui renouvelle le processus de rationalisation de ces activités.

À partir de 1995, le mode de financement des activités de formation des migrants par marchés publics est de plus en plus privilégié (Etienne, 2004 ; Leclercq, 2012 ; Normand \& Sintive, 2012). Le changement des règles de financement a des conséquences, notamment sur les types d'organismes susceptibles d'intervenir pour la formation des migrants et, par conséquent, sur la culture professionnelle.

En effet, les petites associations n'existent souvent que par l'obtention de subventions. Le Haut Conseil à l'Intégration relevait, en 2012, que « le nombre d'associations financées par l'État et oeuvrant expressément dans le domaine de l'intégration des immigrés [... ] a diminué de $80 \%$ » entre 2000 et 2010 (Normand \& Sintive, 2012, p. 6). 
Par ailleurs, à partir des années 2000, les appels d'offres incitent au recrutement de professionnels qualifiés en didactique du FLE (Leclercq, 2012, p. 187) alors que la culture " traditionnelle » du champ est plutôt au recrutement de professionnels aux profils très variés, allant de la cuisinière au juriste (Etienne, 2004 ; Morisse, 2003 ; Vadot, 2014).

Nous pouvons interroger aussi à propos de la façon dont sont formés les formateurs en FLE afin de répondre aux besoins pluridisciplinaires des migrants. Cette analyse du développement historico-culturel des activités de formation des migrants en France peut être approfondie par l'étude des réformes qui ont redéfini le cadre didactique pour ces activités.

\subsection{Réformes du cadre didactique}

À l'échelle européenne, des décisions prises entre la fin des années 1990 et le début des années 2000 ont engagé l'évolution du cadre didactique de la formation des migrants : en 1997, le traité d'Amsterdam prévoit en effet que les politiques migratoires soient gérées d'une manière communautaire (Giugni \& Passy, 2002). Cela a engendré une vague de re-définitions des politiques migratoires dans les différents pays membres (Extramiana \& Van Avermaet, 2010), rendant l'assiduité en formation obligatoire sept fois sur dix (Vincent, 2005), en lien avec l'accès aux premiers titres de séjours (DGLFLF, 2011, p. 79).

En France, le Contrat d'Accueil et d'Intégration (CAI) ${ }^{5}$ a été expérimenté à partir de 2003 puis généralisé en 2006 (DGLFLF, 2011, p. 80). Signé entre le migrant et l'État, ce contrat prévoit plusieurs formations ${ }^{6}$ qui sont commandées aux organismes par l'Office Français pour l'Immigration et l'Intégration ${ }^{7}$ via des marchés publics. Le Diplôme Initial de Langue Française (DILF) a été conçu, en $2006^{8}$, afin de fournir une issue diplômante à la formation linguistique prévue par ce contrat (Beacco, de Ferrari, \& Lhote, 2008, p. 15).

De manière concomitante à la création de ce contrat et du DILF, les utilisations de Technologies de l'Information et de la Communication (TIC) ${ }^{9}$ sont de plus en plus prescrites

5. Nommé Contrat d'Intégration Républicaine à compter du 07/03/16 (consulté de : http://www . ofii . fr/ s_integrer_en_france_47/?sub_menu=5, le 03/08/17)

6. Des formations courtes (d'une journée de $7 \mathrm{~h}$ en général) : une formation civique, une session d'information sur la vie en France, un bilan de compétences professionnelles; et une formation longue, pour l'apprentissage du français, pouvant aller jusqu'à 300 heures pendant un an, renouvelable une fois.

7. Créé en 2009, l'OFII est le "seul opérateur de l'Etat chargé de l'immigration légale » (extrait de l'historique établi sur le site de l'OFII, http://www.ofii.fr/qui_sommes-nous_46/index.html?sub_menu=1, page consultée le 23/03/16)

8. Précédant les six niveaux du Cadre Européen Commun pour les Langues, pensés pour des populations scolarisées, le DILF tient particulièrement compte des migrants peu lettrés (Beacco, de Ferrari et Lhote, 2005, p. 3). Son évaluation repose à $70 \%$ sur l'oral et à $30 \%$ sur l'écrit. En 2013, «61,5\% des bénéficiaires de formation linguistique ont été orientés vers un parcours conduisant au DILF » (DGLFLF, 2013, p. 81).

9. Nous considérons, sous l'acronyme TIC, tout artefact traitant des informations numérisées et susceptible d'être mobilisé pour la formation des migrants : «S'agissant des technologies, il est un point à préciser sans cesse : ce que l'on appelle multimédia (ou TICE ou outil informatique) est en fait un construit extrêmement hétérogène, qui ne donne pas du tout lieu aux mêmes stratégies d'adoption, d'adaptation et d'appropriation »(Baron \& Descartes-Paris, 2005, p. 1). 
au sein des activités de formation (Beauné, 2015). Pour les organismes candidats à la labellisation FLI, par exemple, plusieurs des items du référentiel en lien direct avec les TIC sont présentés comme des «[...] critères [devant] être remplis pour obtenir le Label FLI ${ }^{\circledR}$ ou l'Agrément FLI ${ }^{\circledR} »($ Adami et al., 2011, p. 17), que ces organismes emploient des salariés et/ou des bénévoles.

À l'instar de la définition du FLI, du CAI et du DILF, l'augmentation des prescriptions relatives aux utilisations de TIC pour la formation des migrants qui s'installent en France aujourd'hui peut indiquer une expansion de l'objet des activités de formation des migrants au sens de la théorie de l'apprentissage expansif (Engeström, 1987) : celui-ci évoluerait d'une forme initiale ou traditionnelle (notamment sans utilisation de TIC) vers une forme culturellement plus avancée (du fait, entre autres, des utilisations de TIC).

Face à ce contexte, nous nous sommes donc demandé si les réformes engagées depuis le début des années 2000 en France désignaient une reconnaissance institutionnelle des besoins des migrants et si nous assistions à l'affirmation d'un champ professionnalisé pour la formation de ces publics. Une formulation initiale de notre problématique de recherche peut être formulée ainsi : peut-on constater, au sens de la théorie de l'apprentissage expansif, une expansion de l'objet de ces activités? Dans la partie suivante, nous précisons le cadre de référence mobilisé pour répondre à ce questionnement.

\section{Cadre de référence}

\subsection{Théorie de l'apprentissage expansif}

Nous nous sommes appuyée sur la théorie de l'apprentissage expansif (Engeström, 1987) qui s'inscrit dans la continuité des théories de l'activité (Leontiev \& Cole, 2009 ; Rubinstein, 2007 ; Vygotsky, 1985). Ces théories se sont développées depuis le début du XXe siècle, notamment dans le cadre des travaux russes de psychologie du développement. Les travaux de Leontiev ont permis, entre autres, de distinguer le but des actions individuelles, des mobiles ou de l'objet d'une activité humaine collective (Leontiev \& Cole, 2009, p. 187).

La théorie de l'apprentissage expansif synthétise et développe les modèles du développement humain qui la précèdent, en revendiquant le caractère systémique des relations entre les différents pôles de tout système d'activité humaine, à savoir : le sujet qui agit au sein d'une communauté, dont l'activité est organisée par des règles et la division du travail, et qui mobilise des instruments pour remplir, avec d'autres, l'objet de l'activité collective (Engeström, 1987, p. 94).

Des difficultés, des tensions émergent quotidiennement au cours des activités : elles sont les indicateurs de contradictions dans le système d'activité collectif et les sources de l'expansion de l'activité (Engeström, 1987, p. 97). La première des contradictions internes

à l'activité humaine renvoie à sa dualité intrinsèque : la vie de chaque individu constitue une activité indépendante, simultanément subordonnée à l'activité sociale totale. 
Cette contradiction prend ses sources dans la division sociale du travail et renvoie notamment à la régulation des relations entre la valeur d'échange et la valeur d'usage des services et produits des activités humaines :

La valeur d'échange des biens est essentiellement déterminée par la quantité moyenne de travail social nécessaire à sa production. La contradiction essentielle consiste alors dans l'exclusion et la dépendance, simultanées et mutuelles, de la valeur d'usage et de la valeur d'échange. Cette double nature et cette agitation intérieure caractérisent tous les angles de la structure triangulaire de l'activité (Engeström, 2011b, p. 172).

L'analyse des contradictions permet de comprendre les processus à l'œuvre dans l'évolution de systèmes d'activité, sa mise en oeuvre nous permettait de répondre à la problématique initialement formulée, relative à l'expansion de l'objet de l'activité de formation des migrants.

Les analyses des contradictions peuvent aussi être mises au service d'une méthodologie de recherche développementale et interventionniste, dite des « laboratoires du changement » (Engeström, 2007). Cela dit, l'utilisation des concepts et modèles n'impose pas celle de la méthodologie des laboratoires du changement.

\subsection{Méthodologie des laboratoires du changement}

Cette méthodologie exige la création d'un partenariat explicite entre une équipe de chercheurs et les membres d'une communauté donnée, souhaitant résoudre un problème, une difficulté initiale. Suite à la détermination du partenariat, les chercheurs choisissent et soumettent aux acteurs de la communauté, une sélection de données filmées, permettant de cerner de potentielles contradictions dans le système d'activité.

Ces interventions visent à aider la communauté à élaborer, via l'échange collectif, des solutions créatives pour dépasser les contradictions :

Celles-ci ne peuvent être dépassées qu'en travaillant sur un troisième niveau : celui qui dépasse les deux pôles en opposition. Quelque chose comme une totalité, ouvrant de nouvelles possibilités pour un nouveau niveau de développement de la totalité, et non pas seulement une façon d'équilibrer deux éléments en opposition. Ce n'est pas un mouvement d'équilibration, ni une addition de deux termes, encore moins un compromis ou un amalgame : c'est un dépassement (Engeström, 2011b, p. 172).

La méthodologie des laboratoires du changement guide l'élaboration d'interventions formatives (Barma, Laferrière, \& Allaire, 2013 ; Engeström, 2011a) visant le dépassement créatif des contradictions, via l'expansion de l'objet ou la re-détermination collective du 
mobile de l'activité. On peut dire que ces interventions se distinguent des recherchesactions, en ce qu'elles « [produisent] des savoirs pratiques, dont on peut saisir les retombées sociales » (Wittorski \& Roquet, 2013).

Ce cadre théorique entre en correspondance avec la posture de recherche que nous souhaitons élaborer, avec notre intention d'étudier l'évolution des activités de formation des migrants et de développer des interventions visant à soutenir leur développement. Mais nous avons aussi, dès le début de la thèse, cherché à mobiliser un cadre de référence pluriel et travaillé à articuler différentes conceptualisations concernant les pratiques de formation instrumentées, notamment avec des TIC. Nous avons développé une démarche exploratoire vis-à-vis des instruments de recherche disponibles, dans une volonté d'enrichissement de notre culture scientifique.

Les adaptations de la méthodologie des laboratoires du changement que nous avons élaboré ont aussi, par ailleurs, répondu aux spécificités des terrains d'intervention : en effet, même si les équipes pédagogiques des organismes où nous avons développé notre recherche étaient d'accord pour coopérer dans le cadre d'une démarche interactive et développementale de recherche, nous n'avons pas pu filmer les activités observées : les formateurs s'y sont généralement opposés parce que, pour eux, les apprenants migrants, notamment ceux peu ou non scolarisés, risquaient de ressentir de l'insécurité.

Et, alors qu'il convient d'intervenir avec une équipe de chercheurs, nous intervenions seule sur les terrains : nous avons donc élaboré des adaptations dont nous pensons qu'elles peuvent intéresser des chercheurs, également contraints dans la mise en œuvre d'un tel cadre de référence. Dans la suite, nous présentons les interventions mises en œuvre, à propos desquelles on peut dire qu'elles correspondent à des formes « faibles »d'interventions formatives, inspirées par la méthodologie des laboratoires du changement.

\section{Méthodologie de collecte, d'utilisation et d'analyse des données}

\subsection{Collecte des données}

Des monographies préliminaires à propos de chacun des trois organismes ont d'abord été rédigées, sur la base d'entretiens exploratoires et de recherches complémentaires à propos des organismes, menées en ligne. Nous avons ensuite mené des cycles d'observations participantes (Winkin, 1997) pendant un an pour le premier des organismes et pendant six mois pour les deux autres.

Les trois organismes considérés dans ma recherche étaient tous des centres répertoriés pour la passation du DILF dans une des dix-huit régions françaises :

- le premier organisme de formation participant correspondait à une association subventionnée, notamment par la municipalité et le FSE (Fonds Social Européen); 
- les deux autres correspondaient à des organismes publics de formation continue, tous deux engagés dans le marché public du CAI mais en fonction de durées différentes : le premier de ces deux organismes publics s'y est engagé quand nous avons commencé la recherche de terrain avec eux, en 2013, alors que le second était engagé sur ce marché depuis sa création en 2006.

Ces trois terrains présentaient des contrastes intéressants pour une recherche qualitative : par le type, par la taille des organismes, par leur offre de formation, par leur histoire et leur culture éducative, etc.

Le premier cycle d'observation s'ouvrait avec la rencontre des responsables des organismes visant à leur présenter les questions et les méthodes de notre recherche. Une fois leur accord obtenu pour la poursuite de la recherche avec leur équipe, des entretiens de groupes focalisés (Thibeault, 2010) ont été menés avec les équipes de formateurs.

Suite aux entretiens de groupe et en fonction de la taille des équipes, de l'organisation des cours (durée, fréquence) et de la situation géographique des différents organismes, nous avons planifié des séances d'observation en veillant à aménager une certaine régularité afin que les formateurs, comme les apprenants, puissent s'habituer à ma présence. Des analyses itératives des contradictions ont été réalisées à partir de chaque observation. Des synthèses de ces analyses ont ensuite été périodiquement soumises à la discussion collective.

Des entretiens semi-directifs individuels ont également été menés au cours des observations pour reformuler l'analyse des contradictions et l'identification de leviers pour le développement des activités. Il s'agissait, à chaque fois, d'échanger avec les différents acteurs au sujet de questions ayant émergé au cours des observations. En général, nous avons pris note de tous les événements susceptibles d'enrichir les monographies et survenus à différents moments de contact avec les acteurs de ces organismes.

\subsection{Adaptations de la méthodologie des laboratoires du changement mises en oeuvre}

Les adaptations de la méthodologie des laboratoires du changement que nous avons développées ont donc d'abord concerné la méthodologie d'observation : comme nous n'avons pas pu filmer, nous avons procédé à des prises de notes systématiques en rapport avec les pôles de tout système d'activité et les différentes contradictions (cf. p.4).

Une autre adaptation importante a été constituée par l'envoi périodique, aux acteurs de terrain, de synthèses problématisées à partir des notes d'observation, ces envois étant suivis de discussions collectives ${ }^{10}$.

10. Il est arrivé, au cours d'une de ces discussions, qu'on identifie des tensions interindividuelles fortes : nous avons proposé à la personne qui avait réagi particulièrement vivement au cours des échanges de re-discuter de la situation en entretien individuel. A posteriori, cet entretien a largement permis d'approfondir la première analyse des contradictions. On suppose qu'il a aussi permis à la personne de se sentir écoutée, voire soutenue dans l'expression des difficultés auxquelles elle faisait face. 
Ces propositions d'adaptation de la méthodologie des laboratoires du changement sont en cours de raffinement dans le cadre du projet ReVEA (Ressources Vivantes pour $1^{\prime}$ Enseignement et l'Apprentissage) ${ }^{11}$ à partir de sessions d'échanges à l'oral, soutenu par des supports écrits préparés, avec des équipes pédagogiques ou des collectifs d'enseignants.

Le cadre de la théorie de l'apprentissage expansif nous a permis d'être attentive aux processus à l'œuvre, il est venu soutenir l'observation et l'analyse des évolutions des systèmes d'activité observés. Les contraintes dans sa mise en œuvre ont influencé nos observations de recherche : ne pouvant organiser de laboratoires du changement au sens strict, nous devions considérer des processus d'évolution spontanés. Une nouvelle série de questions a alors émergé : quelles transitions spontanées sont observables? Comment caractériser le développement spontané des systèmes d'activité observés? Comment s'organise le changement spontané lorsqu'il est faiblement guidé?

Quelques conjectures peuvent être énoncées de la manière suivante : le développement spontané d'un système d'activité serait similaire à celui qui peut se produire dans le cadre d'un partenariat explicite avec une équipe de chercheurs mais les résultats différeraient, ils pourraient être moins élaborés ou moins résistants à la diffusion et à la généralisation, moteur de l'expansion de l'objet des activités.

Dans la partie suivante, nous présentons les principaux résultats des analyses des processus d'évolution spontanée, à la fois à travers l'étude de la littérature disponible au sujet des activités de formation des migrants et celle des contradictions soumises à la discussion au sein des trois organismes de formation où nous sommes intervenue.

\section{Principaux résultats}

\subsection{Enjeux et contradictions pour les activités de formation des migrants en France}

L'enjeu fondamental des activités de formation des migrants consiste, non pas dans la seule intégration des migrants à la société française, mais bien dans l'intégration de la société à elle-même (Schnapper, 2007).

Face à cet enjeu et, entre autres résultats, une analyse de contenus de la littérature disponible à propos des activités de formation des migrants a permis d'identifier un nombre important de contradictions (Beauné, 2015, pp. 178-184). Ces contradictions ont également été identifiées dans les organismes de formation où nous avons mené nos observations.

Nous avons pu, entre autres, caractériser l'état de besoin de transformation de ces activités, à partir de l'identification des contradictions suivantes :

11. Soutenu par l'Agence Nationale de la Recherche (ANR) pour une durée de 4 ans (2014-2018), le projet ReVEA (Ressources Vivantes pour l'Enseignement et l'Apprentissage) s'intéresse aux processus de recherche, de sélection, de classement, de production, de modification, de réutilisation, de suppression et de diffusion 


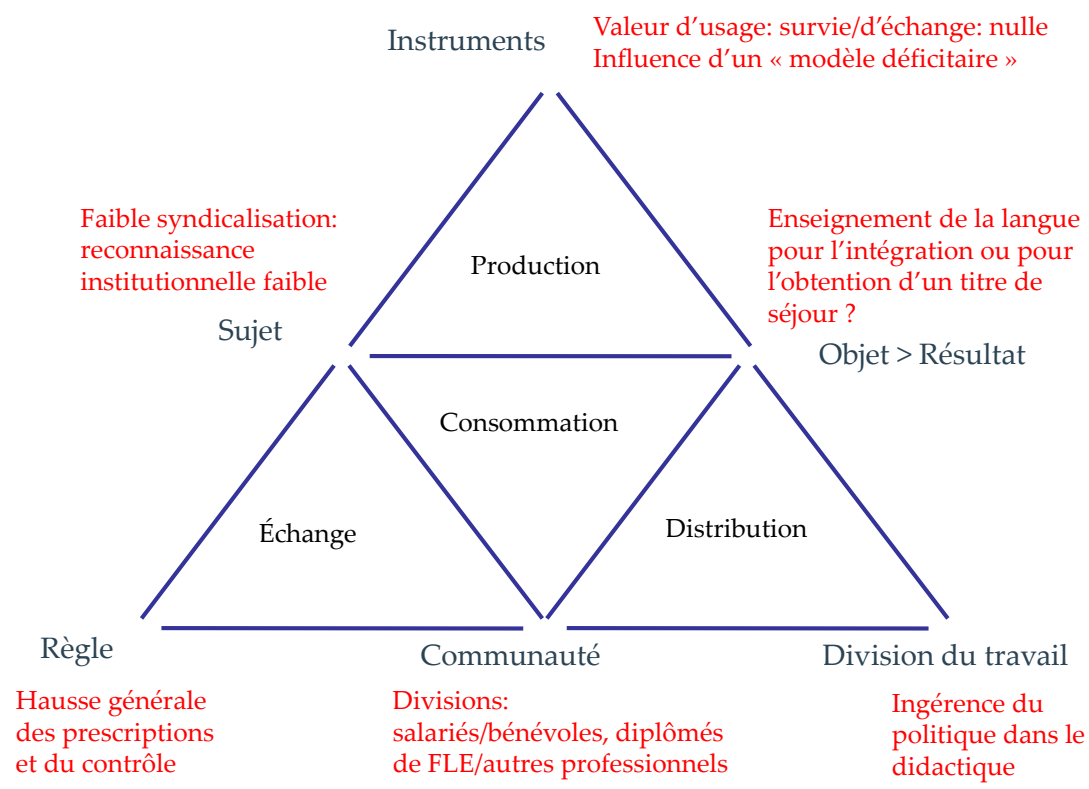

FIGURE 1. Analyse des contradictions primaires pour les activités de formation des migrants (Beauné, 2015, pp. 178-182)

D'autres analyses ont permis d'identifier des contradictions secondaires, prenant la forme d'injonctions paradoxales telles " travaille gratuitement », « intègre-les seul », etc.

Un résultat important de notre recherche a été identifié particulièrement grâce au processus itératif d'analyse des contradictions, mis en œuvre au cours des observations dans les trois organismes de formation. Nous le présentons dans la suite, en tâchant de mettre en évidence les relations entre ce résultat, les interventions inspirées de la méthodologie des laboratoires du changement présentées plus haut et l'analyse des évolutions ou transitions spontanées au sein des systèmes d'activité.

\subsection{Transitions expansives?}

L'analyse itérative des contradictions observées au sein des trois organismes de formation est venue interroger un autre modèle conçu pour guider les interventions formatives des laboratoires du changement (Engeström, 1987, p. 228) : ce modèle présente les différentes transitions expansives entre quatre types historiques d'activité humaine. On en propose ici une traduction qui a un caractère interprétatif :

de ressources1, mis en œuvre par les enseignants dans quatre disciplines pour le secondaire en France : les mathématiques, l'anglais, la physique-chimie, les Sciences et Technologies Industrielles (STI). 


\section{Activité artisanale}

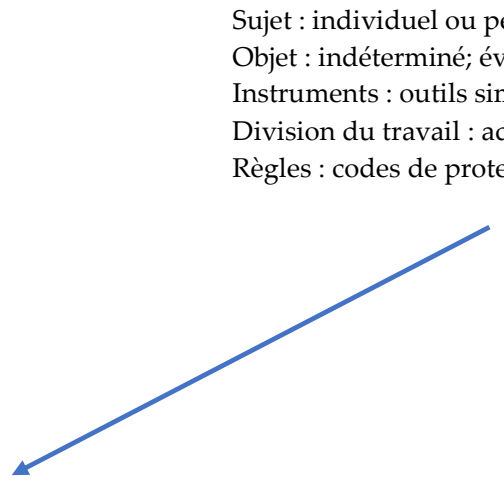

Sujet : individuel ou petit groupe.

Division du travail : adaptation individuelle.

\section{Activité rationalisée}

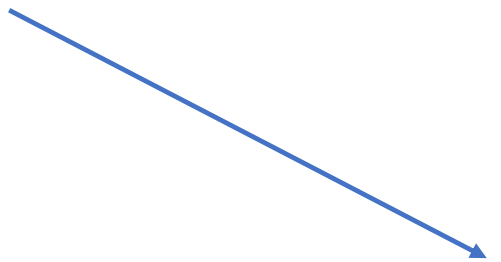

Activité humanisée
Sujet : cadres (management).

Objet : prédéterminés.

Instruments : prédéterminées : machines

complexes, savoir-faire implicites.

Division du travail : hiérarchisée et

compartimentée.

Règles : codes de compétition et de contrôle.
Sujet : cadres et groupes semi-autonomes. Objet : et instruments : comme dans le type rationalisé.

Division du travail : groupes semi-autonomes comme unités de production.

Règles : coopération et communication intragroupe; compétition et contrôle entre groupes.

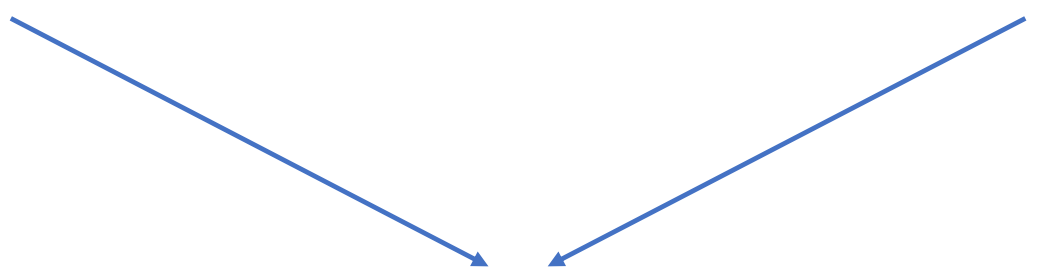

Activité maîtrisée collectivement

dans une perspective expansive

Sujet : collectif.

Objet : l'activité elle-même comme système.

Instruments : secondaires : tremplin, modèles,

microcosme; tertiaires : méthodologie de

développement du travail.

Division du travail : groupes flexibles et

combinaison de tâches.

Règles : comme dans le type humanisé mais

étendu à l'ensemble de l'activité.

FIGURE 2. Quatre types historiques d'activité (Beauné \& Baron, 2014) 
Les quatre types (artisanal, rationalisé, humanisé, maîtrisé collectivement dans une perspective expansive) renvoient aux différents stades identifiés dans l'évolution historique et culturelle des activités humaines. Ils renvoient aussi à ce pour quoi la méthodologie des laboratoires du changement a été conçue : en effet, cette méthodologie vise à accompagner les organisations pour qu'elles atteignent le quatrième type d'activité, celle dite " maîtrisée collectivement dans une perspective expansive ". Au sein de ce type d'activité, les sujets mobilisent volontairement des tremplins, des modèles et des microcosmes pour re-déterminer l'objet de leur système et, partant, l'ensemble de leur activité collective.

Dans le modèle ci-dessus, l'orientation est assez clairement marquée vers le quatrième type historique d'activité, dès lors présenté comme le type historique le plus avancé. On peut considérer que l'orientation donnée aux principaux constituants du modèle (le type artisanal puis celui rationalisé, etc.) est indicative, c'est-à-dire que chacun des types historiques constitue un attracteur en fonction duquel on peut situer les systèmes d'activité observés dans une trajectoire développementale. Cela reviendrait à dire, à partir des données collectées, qu'un certain système relève plutôt du type artisanal ou plutôt du type humanisé, etc.

Il est apparu que cette formulation, qui désigne une conceptualisation (notamment via le « ou » logique), peut entrer en tension avec les données recueillies via l'observation de l'activité d'une communauté donnée. Confrontée aux données que nous avons recueillies, nous avons en effet pu identifier, de manière simultanée et pour chacun des organismes de formation, différentes caractéristiques des différents types du modèle.

Une fois finalisée, l'analyse permettait d'identifier des similarités entre les situations au sein des trois organismes de formation. Ces similarités pouvaient être associées à une trajectoire de transition spontanée (et faiblement guidée) qui n'apparaît pas dans le modèle précédemment montré, ne serait-ce parce que les flèches sont la plupart du temps unidirectionnelles : nous avons identifié par là ce qu'on a choisi de nommer une transition « restrictive ».

À la différence d'une transition expansive (ou d'une redéfinition qualitative de l'objet des activités), une transition restrictive correspondrait au produit d'une interrelation forte entre le type artisanal et le type rationalisé du modèle précédent. Cette interrelation était importante et manifeste, par rapport aux relations identifiées entre la situation des organismes de formation et les autres types du modèle. Une transition restrictive produirait a minima par une limitation ou une restriction de l'objet des activités du fait de cette interrelation privilégiée entre le type artisanal et le type rationalisé.

\subsection{Fondements et limites dans la considération de ces transitions spontanées}

Nous avons bien conscience qu'une fonction du modèle des types historiques d'activité (cf. illustration 2) est de représenter le résultat attendu de l'utilisation de la méthodologie des laboratoires du changement. En cela, il constitue un instrument pour clarifier les mobiles 
du chercheur qui souhaite soutenir le développement des activités observées. Il est toutefois aussi reconnu que les transitions développementales ne suppriment pas leur héritage :

Les fonctions mentales inférieures ne disparaissent pas d'une psyché mûre mais elles sont structurées et organisées en fonction des moyens de production et des buts sociaux spécifiquement humains (Kozulin, 1986, p. 103, traduction libre).

Face à nos données et forte de ce dernier constat, il a semblé nécessaire de proposer des outils pour concevoir la coexistence des différents types historiques au sein d'un même système d'activité. Dans un second temps, ces instruments devraient soutenir la réflexion à propos de transitions différentes de celles allant d'un type initial à celui "plus avancé ", dans un déroulement idéal du développement.

Nous avons proposé, tout d'abord, d'utiliser la notion de dimensions structurelles plutôt que celle de " types historiques ". En effet, la notion de type renvoie à un " moule, modèle idéal qui détermine la forme d'une série d'objets qui en dérivent » ${ }^{12}$ : cette notion peut évoquer un cadre de pensée fordiste en termes de productivité.

En contraste, la notion de «dimension» permet de considérer une «composante spécifique et relativement importante, quoique parfois mal perçue, de la pensée ou du réel» ${ }^{13}$. Cette définition, en "composante spécifique», autorise à considérer la coexistence de différentes dimensions, la simultanéité de leur manifestation.

Une des limites à la proposition d'analyser la coexistence de dimensions structurelles au sein des systèmes d'activité, c'est qu'elle procure un effet d'instantané, comme un arrêt sur image alors que la théorie de l'apprentissage expansif s'intéresse, en premier lieu, aux évolutions en cours, au soutien des dynamiques d'apprentissage.

Cet effet d'instantané peut toutefois aider la conceptualisation, pour les chercheurs impliqués, de tensions importantes pour les systèmes d'activités observés. Notre proposition prend, en ce sens, un caractère méthodologique plus que théorique. Par ailleurs, elle ne vient pas pour annuler et remplacer le modèle précédent mais plutôt en complément, dans le sens où elle invite à considérer des transitions qui n'y apparaissent pas.

\subsection{Analyse de transitions restrictives dans les trois organismes de formation}

En analysant la coexistence des dimensions structurelles de l'activité des trois organismes de formation, nous avons mis au jour un résultat commun au sujet de l'évolution spontanée (même si elle était faiblement guidée) de leur activité : l'interrelation privilégiée entre les dimensions artisanales et rationalisées engendrait une transition restrictive de l'objet des activités de formation des migrants, prenant particulièrement ses sources dans les règles de financement.

12. Acception consultée le 27/03/16, de : http://www . cnrtl.fr/lexicographie/type

13. Acception consultée le $27 / 03 / 16$, de : http://www . cnrtl. fr/lexicographie/dimension 
Dans le cas de l'association, l'analyse des contradictions a en effet montré que les contraintes de plus en lourdes pour l'obtention des subventions augmentaient la dimension rationalisée de leur activité mais, comme il s'agit de subventions, donc de financements peu pérennes, cela entretenait la dimension artisanale des activités de formation.

La prédétermination de l'objet par les subventions avait pour effet de toucher moins de personnes, pour des durées de plus en plus courtes et sur des axes de formation déformant les besoins détectés par les acteurs de terrain. Les personnes avaient, de ces faits conjugués, de plus en plus de difficultés à déterminer un projet collectif en lien avec les besoins des populations qu'ils cherchent à soutenir pour leur intégration. Les changements du cadre institutionnel et didactique ne favorisaient pas de transition expansive spontanée dans ce contexte.

Dans le cas du premier organisme public, l'analyse des contradictions a montré entre autres que le recours aux appels d'offres a augmenté la concurrence entre organismes et donc les dimensions rationalisées de leur activité. Simultanément, ce recours aux appels d'offres a généré une baisse des coûts d'investissements ${ }^{14}$, maintenant de ce fait certaines dimensions artisanales pour la formation des migrants.

Là aussi, il était difficile de décrire une transition spontanée à caractère expansif. Au contraire, l'interrelation privilégiée entre les dimensions artisanales et rationalisées semblait produire une restriction de l'objet des activités à la délivrance formelle d'heures de formation pour des flux de population dont les besoins étaient faiblement considérés.

Dans le cas du second organisme public, les pratiques des formateurs mettaient l'accent sur l'entraînement des compétences orales au détriment de celles écrites, ce qui attestait un certain consensus à propos de l'objet prédéterminé par le marché public et donc, des dimensions rationalisées du système d'activité. Cependant les supports écrits utilisés, parmi lesquels ceux conçus pour la préparation au DILF, mettaient les apprenants les moins avancés en difficulté et nécessitaient donc des ajustements assez artisanaux de la part des formateurs vis-à-vis de ces apprenants.

Dans ce contexte, comme dans les deux autres organismes de formation où nous avons pu faire des observations et même si l'expérience dans le marché public du CAI était plus importante pour cet organisme, nous ne pouvions pas décrire de transition expansive spontanée : l'activité observée témoignait plutôt de la restriction de l'objet, notamment en ce qui concerne la possibilité de prendre en compte les besoins à l'écrit des apprenants. Or, il est connu depuis les années 1970 que retarder la prise en compte des besoins à l'écrit peut engendrer des sorties de formation (Poilroux \& Colombier, 1974, p. 323).

14. La chute du nombre d'heures de formations prescrites aux apprenants (passant de $1400 \mathrm{~h}$ à 400 puis, à partir de 2016, à 200 heures par an et par personne, voir Etienne (2004, p. 26), liée à l'augmentation de la concurrence entre organismes de formation face aux marchés publics (c'est-à-dire que, pour paraître performant, il vaut mieux afficher un nombre d'heures inférieur à celui annoncé par d'autres pour l'obtention du même résultat), influence négativement la valeur du taux horaire par apprenant dans le cadre des CAI (déclarations relevées à deux occasions lors des observations de terrain). 
L'analyse des interactions entre les dimensions structurelles des systèmes d'activité considérés dans notre recherche tend à montrer qu'une interrelation privilégiée entre dimensions artisanales et dimensions rationalisées peut engendrer des transitions restrictives pour les systèmes d'activité. En ce qui concerne les activités de formation des migrants et dans les trois organismes où nous avons pu faire des observations, nous avons constaté que l'objet de ces activités tend à se restreindre à une formation rudimentaire, uniquement au français, à l'oral, où les TIC sont très peu utilisées, ignorant de ce fait un certain nombre de travaux menés depuis les années 1960 à propos des besoins pluridisciplinaires des migrants.

\section{Synthèse des résultats}

Un premier questionnement pour cette contribution a été formulé ainsi : suite à une importante vague de réformes, comment évoluent les activités de formation des migrants? Ces réformes indiquent-elles une reconnaissance institutionnelle des besoins des migrants via l'affirmation d'un champ professionnalisé pour leur formation? Peut-on constater, au sens de la théorie de l'apprentissage expansif, une expansion de l'objet de ces activités?

L'analyse des données recueillies au cours de notre recherche et particulièrement, le processus itératif mis en œuvre pour l'analyse des contradictions et des transitions spontanées, ont plutôt montré que la rationalisation des activités de formation des migrants se développe à travers les dernières réformes, entrant en tension avec les dimensions artisanales historiques de ces activités.

Ce processus a pour effet de restreindre l'objet des activités à une formation linguistique (plutôt que de l'étendre à une formation pluridisciplinaire ou à des parcours de formation organisant l'intégration) visant l'obtention d'un niveau rudimentaire en français parlé (et limitant explicitement la prise en compte des besoins relatifs au développement de compétences en littéracies) ou celle d'un titre de séjour, voire au « tri » des populations migrantes accueillies sur le territoire.

Les adaptations de la méthodologie des laboratoires du changement ont permis de formuler un nouveau questionnement : quelles transitions spontanées sont observables au moyen d'une observation régulière des activités? Comment caractériser le développement spontané des systèmes d'activité? Comment s'organise le changement spontané lorsqu'il est faiblement guidé?

En ce qui concerne le développement spontané des systèmes d'activité observés, nos résultats tendent à montrer que dans des contextes où peu de concertations collectives sont organisées ou possibles, le développement et l'expansion de l'objet des activités sont difficiles ; les résultats des apprentissages expansifs individuels enrichissent exceptionnellement le patrimoine collectif de résolution des problèmes, tensions ou difficultés rencontrées par les acteurs du système. 


\section{Discussion et perspectives de recherche}

La recherche que nous avons menée a un caractère qualitatif marqué : elle repose sur de petits échantillons qui n'ambitionnaient aucune représentativité. C'est par l'analyse itérative des contradictions ${ }^{15}$, que nous avons pu identifier des transitions spontanées restrictives, fondée sur une interrelation privilégiée entre les dimensions artisanales et rationalisées. Dans les organismes de formation où nous avons mené nos observations, ces transitions spontanées contraignaient fortement la re-détermination de l'objet de l'activité par les acteurs de terrain.

Cela dit, la proposition de la notion de transition spontanée restrictive est encore tout à fait exploratoire et largement à définir, via d'autres analyses de données, pour des terrains ou des pratiques contrastées, de façon à caractériser sa consistance et son potentiel analytique. Il en va de même avec celle de « dimensions structurelles coexistant dans tout système d'activité ».

Nos propositions relèvent d'une réflexion méthodologique en cours : nous cherchons actuellement à les mettre à l'épreuve sur d'autres terrains mais aussi à les soumettre à la communauté pour entretenir une réflexion collective à leur sujet.

Si le caractère de simultanéité entre les dimensions structurelles de tout système d'activité est important dans les propositions faites ici ${ }^{16}$, nous n'avons pas cherché à réduire l'intérêt de la dynamique temporelle telle qu'elle est conçue dans la théorie de l'apprentissage expansif : par exemple, via la centration sur l'analyse des développements historiques et culturels des activités humaines et la méthodologie de soutien des processus de développements à l'œuvre

Un autre terme que celui de «dimension » pourrait être plus adéquat, qu'il entretienne des rapports de synonymie ou non avec celui-ci. Celui de " perspective » (artisanale, humanisée, rationalisée ou maîtrisée collectivement dans une dynamique expansive) pourrait rétablir le caractère dynamique des processus analysés et des interventions formatives conçues pour le développement des systèmes d'activité.

Les propositions faites ici interviennent résolument en complément de l'existant : elles visent à rendre compte des « bouleversements, changements soudains et reprises » ${ }^{17}$ du développement humain (Kozulin, 1986, p. 103). Finalement, une perspective vive se concentre dans le développement de compétences plus avancées en termes d'accompagnement de trajectoires d'innovation pour les formateurs et les équipes pédagogiques.

15. Celles identifiées via l'analyse de contenu de la littérature disponible à propos des activités de formation des migrants et celles identifiées et discutées collectivement au cours des observations dans les trois organismes de formation dans lesquels nous avons pu développer notre recherche.

16. Elles essayent en effet d'apporter des éléments de réflexion pour penser le rapport à l'héritage, aux traditions d'activité.

17. Traduction libre de "Vygotsky perceived psychological development as a dynamic process full of upheavals, sudden changes, and reversals. This process, however, ultimately leads to the formation of the cultural, higher mental functions ». 
Si nous avons interrogé les conceptualisations de la "professionnalisation » dans les recherches en éducation (Beauné, 2015, pp. 149-178) et ré-engagé des actions de terrain afin d'améliorer les propositions d'adaptation de la méthodologie des laboratoires du changement ${ }^{18}$, le " développement professionnel » des enseignants, tel qu'il est conçu pour les Écoles Éloignées en Réseau (Hamel, Laferrière, Turcotte, \& Allaire, 2014) semble prometteur afin d'enrichir notre approche des terrains et de l'accompagnement.

\section{Références}

Adami, H., Bergère, A., Étienne, S., Lambert, P., Poirrier, G., Verdier, C., \& Vicher, A. (2011). Référentiel fli, français langue d'intégration. Ecrimed : DAIC.

Barma, S., Laferrière, T., \& Allaire, S. (2013). Proposition de lexique. Revue internationale du CRIRES : innover dans la tradition de Vygotsky, 1(1), 50-51.

Baron, G.-L., \& Descartes-Paris, R. (2005). Les tice, de l'innovation à la scolarisation : problèmes et perspectives. Congrès national de l'AFT-RN, 25.

Beacco, J.-C., de Ferrari, M., \& Lhote, G. (2008). Niveau A1. 1 pour le français : publics adultes peu francophones, scolarisés, peu ou non scolarisés; référentiel et certification (dilf) pour les premiers acquis en français. Paris : Didier, Conseil de l'Europe et Division des politiques linguistiques.

Beauné, A. (2015). Contribution à l'étude des pratiques instrumentées des formateurs d'adultes : le cas $d u$ dilf (Thèse de doctorat, Université Paris 5 Sorbonne Descartes). https:// hal . archives-ouvertes . fr/tel-01401880.

Beauné, A., \& Baron, G.-L. (2014). Les activités associatives visant l'intégration des migrants dans un contexte institutionnel changeant. Savoirs et Formation, Recherches et Pratiques, $4,58-74$.

De Lescure, E. (2004). La construction du système français de formation professionnelle continue. retour sur l'accord du 9 juillet 1970 et la loi du 16 juillet 1971. Paris : L'Harmattan.

DGLFLF. (2011). Rapport au parlement sur l'emploi de la langue française. http://www. culturecommunication.gouv.fr/content/download/93626/ 841497/version/3/file/rapport_parl_2011_def.pdf.

DGLFLF. (2013). Rapport au parlement sur l'emploi de la langue française. http: //www . langue -francaise.org/rapport_DGLF_2013.pdf.

Engeström, Y. (1987). Learning by expanding : an activity-theoretical approach to developmental research. Helsinki : Orienta-Konsultit Oy.

Engeström, Y. (2007). Putting Vygotsky to work : The change laboratory as an application of double stimulation. In The cambridge companion to Vygotsky (pp. 363-382). New York : Cambridge University Press.

Engeström, Y. (2011a). From design experiments to formative interventions. Theory $\mathcal{E}$ Psychology, 21(5), 598-628.

Engeström, Y. (2011b). Théorie de l'activité et management. Management $\mathcal{E}$ Avenir(2), $170-182$.

Etienne, S. (2004). Pour une prise en compte d'un secteur négligé en didactique du français langue étrangère : la formation de base (Thèse de doctorat, Université de la Méditerranée-AixMarseille II). http: //tel . archives-ouvertes . fr/tel-00151868/.

18. Dans le cadre du projet ReVEA avec le suivi d'un collectif d'enseignants. 
Extramiana, C., \& Van Avermaet, P. (2010). Enquête sur l'intégration linguistique des migrants adultes dans les etats membres du conseil del'europe. In Conference of the council of europe on'linguistic integration of adult migrants'. (Vol. 24, p. 25). Strasbourg. http:// www . coe.int/t/dg4/linguistic/Source/ConfMigr10_C-Extramiana-text.doc.

Giugni, M., \& Passy, F. (2002). Chapitre 11. In Le champ politique de l'immigration en europe: Opportunités, mobilisations et héritages de l'etat national (pp. 433-460). Paris : Presses de la Fondation des sciences politiques.

Hamel, C., Laferrière, T., Turcotte, S., \& Allaire, S. (2014). Un regard rétrospectif sur le développement professionnel des enseignants dans le modèle de l'école éloignée en réseau. STICEF, 20. http://sticef.univ-lemans.fr/num/vol2013/20-hamel -cren/sticef_2013_NS_hamel_20.htm.

Kozulin, A. (1986). The concept of activity in soviet psychology : Vygotsky, his disciples and critics. American psychologist, 41(3), 264.

Leclercq, V. (2012). La formation des migrants en france depuis l'alphabétisation des années 1960. In Les migrants face aux langues des pays d'accueil (pp. 173-196). Villeneuve d'Ascq : Presses du Septentrion.

Leontiev, A. N., \& Cole, M. (2009). The development of mind : Selected works. Pacifica, CA : Marxists Internet Archive.

Morisse, M. (2003). Analyse des besoins de formation des formateurs intervenant dans les actions de formation linguistique et/ou de base en région provencealpes-côte d'azur. CLP, CRI PACA. http://www.illettrisme.org/components/ com_remository_files/downloads/163_Morisse.pdf.

Normand, B., \& Sintive, J.-C. (2012). Investir dans les associations pour réussir l'intégration. HCI (Haut Conseil à l'Intégration. http: //archives . hci . gouv . fr/IMG/pdf/ Avis_association.pdf.

Poilroux, J., \& Colombier, P. (1974). Présentation, explication, répétition, exploitation. les travailleurs migrants et le moteur sgav à quatre temps. In Actes du troisième colloque international sgav pour l'enseignement des langues (pp. 321-326). Châtenay-Malabry, France : CREDIF.

Puren, C. (2014). La revue de presse du fli, français langue d'intégration. http:// www . christianpuren. com/fli/.

Rubinstein, S. L. (2007). Fondements de la théorie de la conscience. In Rubinstein aujourd'hui. nouvelles figures de l'activité humaine (pp. 241-253). Toulouse : Octarès Editions.

Schnapper, D. (2007). Qu'est-ce que l'intégration? Paris : Gallimard.

Thibeault, E.-N. (2010). A propos de la méthodologie des entretiens de groupe focalisés. Adjectif. http://www . adjectif.net/spip/spip.php?article58.

Vadot, M. (2014). Les conditions de travail des formateurs et formatrices dans le contexte actuel de la formation de base. Savoirs et Formation-Recherches et Pratiques, 4, 104-124.

Vincent, D. (2005). La formation des étrangers à la langue du pays d'accueil (etude de législation comparée no. 150). http://www. senat.fr/lc/lc150/lc150_mono.html.

Vygotsky, L. S. (1985). Pensée et langage, trad. F. Sève. Paris : Éditions sociales.

Winkin, Y. (1997). L'observation participante est-elle un leurre? Communication et organisa$\operatorname{tion}(12)$.

Wittorski, R., \& Roquet, P. (2013). Professionnalisation et déprofessionnalisation : des liens consubstantiels. Recherche E formation(1), 71-88. 